\begin{tabular}{|c|c|c|}
\hline $\begin{array}{l}\text { EXCELLENT } \\
\text { PUBLISHERS } \\
\end{array}$ & $\begin{array}{c}\text { International Journal of Current Research } \\
\text { and Academic Review } \\
\text { ISSN: 2347-3215 (Online):;:Volume 5;,;Number } 1 \text { (January-2017) } \\
\text { Journal homepage: http://www.jicrar.com }\end{array}$ & 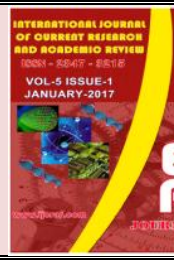 \\
\hline
\end{tabular}

doi: http://dx.doi.org/10.20546/ijcrar.2017.501.007

\title{
Awareness of Oral Health among Lawyers and Law College Students- A Questionnaire Study
}

\author{
Muthu Laakshmi Ganesh ${ }^{1 *}$ and Jai Ganesh Ramamurthy ${ }^{2}$ \\ ${ }^{1}$ Undergraduate Student, Saveetha Dental College, Chennai, India \\ ${ }^{2}$ Reader, Department of periodontics, Saveetha dental college, Chennai, India \\ *Corresponding author.
}

\begin{tabular}{|c|c|}
\hline Abstract & Article Info \\
\hline \multirow{8}{*}{$\begin{array}{l}\text { To assess the oral health awareness among lawyers and law college students. The } \\
\text { objective of the study is to assess awareness and knowledge about oral health among } \\
\text { lawyers and law college students. Oral diseases have been a persistent public health } \\
\text { problem globally, with almost every individual experiencing poor oral health at least } \\
\text { once in their lifetime. Poor oral hygiene occurring due to increasing plaque and } \\
\text { calculus deposits with increasing age have been reported among children and } \\
\text { adolescents in general population. Oral health affects the general health, well-being and } \\
\text { diminishes the quality of life. Chronic oral infections can pose a risk for diabetes, } \\
\text { cardiovascular diseases like stroke, respiratory diseases. A questionnaire with } 16 \\
\text { structured questions were prepared and distributed among } 20 \text { lawyers and } 100 \text { law } \\
\text { students to obtain the information. The questions were designed to determine the level } \\
\text { of knowledge and awareness about oral health. Lack of awareness among public is the } \\
\text { reason for gingival and periodontal problems. So this study is conducted to assess the } \\
\text { awareness of oral health in professionals. }\end{array}$} & $\begin{array}{l}\text { Accepted: } 05 \text { January } 2017 \\
\text { Available Online: } 20 \text { January } 2017\end{array}$ \\
\hline & Keywords \\
\hline & $\begin{array}{l}\text { Oral hygiene practices, oral hygiene } \\
\text { awareness, Knowledge. }\end{array}$ \\
\hline & \\
\hline & \\
\hline & \\
\hline & \\
\hline & \\
\hline
\end{tabular}

\section{Introduction}

Oral health is an important aspect of general health and wellbeing. Oral diseases are major public health concern due to high prevalence and its impact on quality of life (Vinod et al., 2007). They can affect the most basic human needs, including the ability to eat and drink, swallow, maintain proper nutrition, smile, and communication.

The dental diseases that are commonly seen in the majority of the people are dental caries and periodontal diseases and these diseases are primarily preventable. By maintaining good personal oral hygiene, oral health can be preserved, whereby microbial plaque is removed and prevented from accumulating on teeth and gingiva.Oral hygiene if adopted properly can help get rid of oral disease. Prevention of oral disease can be achieved by optimising the oral health practices in the form of proper tooth brushing, use of dental floss, dental visits at regular intervals, and proper dietary practices (Azodo, 2012). Better knowledge in oral health practices and their attitude are linked to good habits with healthier oral cavity.

The aim of the study is to assess dental health attitude and behavior among lawyers and law college students in Chennai. 


\section{Materials and Methods}

A questionnaire study was conducted among lawyers and law college students attending a private college in chennai. A self constructed 16 close ended questionnaire was distributed to all subject above 18 years of age. The questionnaire included information related to patients name, age, gender and occupation. It was further categorized to evaluate the knowledge, practices and behaviour pattern related to oral health. The completed questionnaire was then analysed statistically.

\section{Results and Discussion}

1) How often do you brush your teeth?

$70 \%$ of students opted once per day and $30 \%$ students opted twice per day (Figure 1).

2) When do you brush your teeth?

$70 \%$ of student opted morning and $30 \%$ students opted both morning and night.

3) For how long do you brush your teeth?

$40 \%$ of students opted less than one minute and $60 \%$ of students opted two minutes.

4) What kind of bristles you usually prefer while selecting tooth brush?

$78 \%$ of students opted hard and $22 \%$ students opted medium.

5) How often do you change your toothbrush?

$10 \%$ of students opted once in 3 months and $90 \%$ opted once in 6 months (Figure 2)

6) Do you use fluoridated toothpaste for brushing?

$92 \%$ of students opted always and $8 \%$ of students opted sometimes (Figure 3)

7) If yes, do you know what is the advantage of using fluoridated tooth paste?

$12 \%$ of students opted removes stains, $20 \%$ of students opted prevent tooth decay and 68\% of students opted removes soft deposits (Figure 4)

8) Are you following any other methods for cleaning teeth other than brushing?

$36 \%$ of students opted yes and $64 \%$ of students opted no (Table 1)

9) If yes, what are the other methods are you following?
$42 \%$ of students opted use of mouthwash, $53 \%$ of students opted tongue cleaning and 5\% students opted all the above (Figure 5)

10) After taking snack between meals, what do you do? $26 \%$ of students opted rinse my mouth with water and $74 \%$ of students opted I do nothing.

11) How often do you visit your dentist?

$10 \%$ of students opted regularly ever 6-12 months $13 \%$ of students opted occasionally ,20\% of students opted whey they have dental pain and $57 \%$ of students opted I have never visited dentist (Figure 6)

12) Have you undergone scaling (teeth cleaning) treatment before?

$34 \%$ of students opted yes and $66 \%$ of students opted No (Table 1)

13) If yes, what is the possible interval you can undergo with that treatment?

$67 \%$ of students opted 6 months and 33\% of students opted 1 year.

14) Are you aware of any oral disease?

$84 \%$ of students opted yes and $16 \%$ of students opted no (Table 1)

15) If yes, what are they?

$46 \%$ of students opted gingivitis, $22 \%$ of students opted periodontitis and $32 \%$ of students opted both gingivitis and periodontitis.

16) Are you aware of the fact that general Health of a person is linked to oral health?

$92 \%$ of students opted yes and $8 \%$ of students opted no (Table 1)

The present study has investigated the awareness of oral health among lawyers and law college students.

It has been observed that oral hygiene has mostly remained as an ignored and unrealized major social problem. Some people are unaware about the relationship between oral hygiene and systemic diseases or disorders. Most diseases show their first appearance through oral signs and symptoms and they remain undiagnosed or untreated because of this missing awareness.

Preventive oral health knowledge, behavior, and its practice are the important ways of keeping our teeth 
healthy. Hence, in this study attempts were made to evaluate preventive oral health knowledge and practice of the population.

In this study $70 \%$ of students brush their teeth once daily and $30 \%$ of students brush their twice daily. $70 \%$ of students brush in the morning and $30 \%$ of students brush both morning and night. The frequency of brushing is linked to oral hygiene. Many studies have shown that less frequent tooth brushing was associated with high probability of having poor oral hygiene (Thangavelu et al., 2014). Kressin et al., evaluated the effect of oral hygiene practices on tooth retention in a longitudinal study with a 26- year follow up. They observed that consistent brushing (more than once a day) resulted in $49 \%$ reduction of risk of tooth loss compared with participants without consistent hygiene habits (Kressin et al., 2003) A recommendation to brush twice daily should be considered (Echeverria et al., 1987).

In our present study $40 \%$ of students brush their teeth in less than one minute and $60 \%$ of students brush their teeth in two minutes.78\% of students use brush with soft bristles and $22 \%$ of students use brush with medium bristles. This increased use of brushes with soft bristles helps to prevent enamel wear, gingival recession and lesion in gingiva.

$10 \%$ of students change their brush once in three months and $90 \%$ of students change their brush once in six months.

In this study $92 \%$ of students always use fluoridated tooth paste for brushing and $8 \%$ of students sometimes use fluoridated tooth paste. $12 \%$ of students think that fluoridated toothpaste will remove stains, $20 \%$ of students think that it will prevent tooth decay and $68 \%$ of students think that it will remove soft deposits. Fluoride is necessary for mineralization of teeth. Many Studies have shown that fluorides prevent and arrest dental caries (McKelvey et al., 2003). In this study there is general lack of awareness about fluoridated tooth paste which plays a very important role in preventing caries. Some studies shows that most of the dentists recommends the use of brush and paste for cleaning the teeth (Keerththana et al., 2013), which shows selection of tooth paste and tooth brush is important for maintaining proper oral hygiene.

In our study $36 \%$ of students are using methods other than brushing for cleaning the teeth and $64 \%$ of students are not using any other methods for cleaning the teeth other than brushing. $42 \%$ of students use mouthwash, $53 \%$ of students are using tongue cleaner and 5\% of students are using mouth wash, tongue cleaners and floss for cleaning the teeth. Mostly they use it to control malodor. Several studies have shown the usefulness of regular dental flossing for removal of inter dental plaque and calculus (Bauroth et al., 2003; Bellamy et al., 1987).

After taking snack between meals $26 \%$ of students wash their mouth with water and $74 \%$ of students do nothing after taking meals. This missing of very basic method of maintaining oral hygiene is an indication of lack of awareness.

In this study, $10 \%$ of students visit dentist regularly every 6-12 months, $13 \%$ of students visits dentist occasionally, $20 \%$ of students visit dentist when they have dental pain and $57 \%$ of students never visited dentist. Missing awareness about the crucial role of regular dental checkups in preventing and detecting dental diseases is an another gap in public education.

Table 1.

\begin{tabular}{|l|l|l|}
\hline & $\mathrm{YES}(\%)$ & $\mathrm{NO}(\%)$ \\
\hline $\begin{array}{l}\text { Are you following any other methods for cleaning teeth other } \\
\text { than brushing? }\end{array}$ & $36 \%$ & $64 \%$ \\
\hline Have you undergone scaling(teeth cleaning) treatment before? & $34 \%$ & $66 \%$ \\
\hline Are you aware of any oral disease? & $84 \%$ & $16 \%$ \\
\hline $\begin{array}{l}\text { Are you aware of the fact that general Health of a person is } \\
\text { linked to oral health? }\end{array}$ & $92 \%$ & $8 \%$ \\
\hline
\end{tabular}




\section{Figure 1}

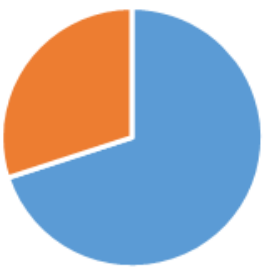

- Once per day $\quad$ Twice per day

\section{Figure 3}

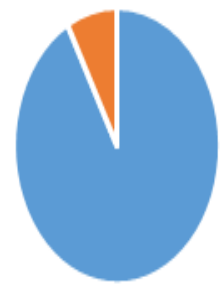

- Yesalways - Sometimes

\section{Figure 5}

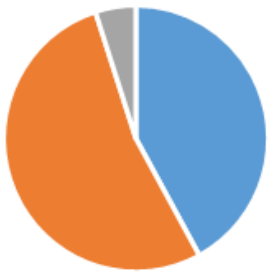

- Use of mouth wash = Tongue cleaning all the above

In this study,34\% of students undergone scaling treatment and $66 \%$ of students have not undergone with scaling treatment.67\% of students think that scaling treatment can be undergone once in 6 months and 33\% of students think that scaling treatment can be undergone once in a year. This shows that awareness was less about dental treatments.
Figure 2

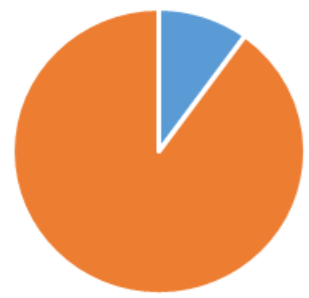

- Once in 3 months $\quad$ Once in 6 months

Figure 4

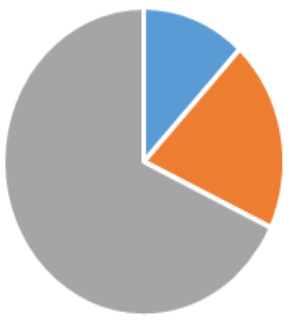

- Removes stains $\quad$ - Prevent tooth decay

- Removes soft deposits

Figure 6

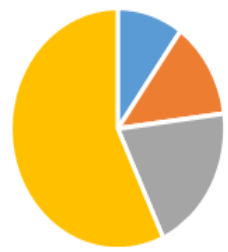

- Regularly every 6-12 months = Occasionally

- When I have dental aim

- I have never visited dentist

In this study $84 \%$ of students were aware of oral diseases and $16 \%$ of students were not aware of any oral diseases. $46 \%$ of students know about gingivitis, $22 \%$ of students know about periodontitis and $32 \%$ of students know about both gingivitis and periodontitis. Most of the students were aware of oral diseases which are common in general population which helps them to prevent further complications. 
In this study $92 \%$ of students aware of the fact that general Health of a person is linked to oral health where $8 \%$ of students were unaware that general Health of a person is linked to oral health. Most of the students in this study were aware that oral health has some influence on general health.

\section{Conclusion}

The results of this study show that awareness about oral hygiene and oral health was quite less among lawyers and law college students. We, as dentists, will have to keep reinforcing the importance of correcting all aspects related with brushing and flossing along with the importance of regular checkups. The task of spreading this awareness extends beyond our clinic to general masses and it will have to be achieved in a similar way by various outreach programs and relevant public health awareness measures through various mediums, such as Print, Press Media, Audio, Radio, Television, Internet, and Organising Social Activities. All of these and more innovative methods of reaching the public will not only ensure a healthy individual but a healthy society as well.

\section{References}

Azodo, C.C., Unamatokpa, B. 2012. Gender difference in oral health perception and practices among Medical House Officers. Russian Open Med. J., 1: 1-4.

Bauroth, K., Charles, C.H., Mankodi, S. M., Simmons, K., Zhao, Q. \& Kumar, L.D. 2003. The efficacy of an essential oil antiseptic mouthrinse vs. dental floss in controlling interproxi-mal gingivitis: a comparative study. J. American Dental Assoc., 134, 359-365.

Bellamy, P., Barlow, A., Puri, G., Wright, K. I.,Mussett, A. \& Zhou, X. 1987. A new in vivo interdental sampling method comparing a daily flossing regime versus a manual brush control. J. Clin. Dentistry, 15: 59-65.

Echeverria, J.J., Manau, C. \& Tejerina, J.M. 1987. [fundamentals of periodontal treatment]. Arch. Odonto Estomatol., 3: 359-364.

Keerththana Balabaskaran, Jaiganesh Ramamurthy. 2013. Assessment of Knowledge of Oral Hygiene Aids among Dentists, IOSR-JDMS, Volume 10, Issue 5: 60-64.

Kressin, et al. 2003. J. Dent. Res., 82(3): 223-227.

McKelvey, V.A., Thomson, W.M. 2003. A qualitative study of oral health knowledge and attitudes among staff caring for older people in Dunedin long-term care facilities. New Zealand Dental J., 99(4): 98103.

Thangavelu, A., Elavarasu, S., Saravanan, J. 2014. Oral health practices and awareness among patients at the department of periodontics at J.K.K.N Dental college and hospital, Komarapalayam. IJCDS, 5(1): 13-6.

Vinod, S., Kamble, Santosh, M., Biradar, Aparna Takpere, Shrinivas Reddy. 2016. Evaluation of oral hygiene awareness and practices among medical students, Int. J. Community Med. Public Health, 3(1): 83-85.

\section{How to cite this article:}

Muthu Laakshmi Ganesh, Jai Ganesh Ramamurthy. 2017. Awareness of Oral Health among Lawyers and Law College Students- A Questionnaire Study. Int.J.Curr.Res.Aca.Rev. 5(1), 61-67.

doi: http://dx.doi.org/10.20546/ijcrar.2017.501.007 


\section{Appendix-1}

\section{Questionnaire}

1) How often do you brush your teeth?
A) less than once per day
B) once per day
C) twice per day
D) more than twice per day

2) When do you brush your teeth?
A)morning
B)noon
C)night before going to bed
D)both morning and night

3) For how long do you brush your teeth?
A)less than one minute
B)two minutes
C)three minutes
D)more than three minutes

4) What kind of bristles you usually prefer while selecting tooth brush?
A)soft
B)ultra soft
C)medium
D) hard

5) How often do you change your toothbrush?
A) once in 2 months
B) once in 3 months
C) once in 6 months
D) never

6) Do you use fluoridated toothpaste for brushing?
A) yes always
B) sometimes
C) rarely
D) never

7) If yes,do you know what is the advantage of using fluoridated tooth paste?
A)removes stains
B)prevent tooth decay

C)removes soft deposits

8) Are you following any other methods for cleaning teeth other than brushing?
A)yes
B)no

9) If yes, what are the other methods are you following?

A)use of mouth wash 

B)flossing
C)tongue cleaning
D)all the above

10) After taking snack between meals, what do you do?
A) brush my teeth immediately
B) I rinse my mouth with mouthwash
C) rinse my mouth with water
D) I do nothing

11) How often do you visit your dentist?
A) regularly every 6-12 months
B) occasionally
C) when I have dental pain
D) I have never visited a dentist

12) Have you undergone scaling(teeth cleaning) treatment before?
A)yes
B)no

13) If yes, what is the possible interval you can undergo with that treatment?
A)6 months
B) 1 year
C) others please specify,

14) Are you aware of any oral disease?
A)yes
B)no

15) If yes, what are they?
A)gingivitis
B)periodontitis
C) Both (A) and (B)
D)others please specify

16) Are you aware of the fact that general Health of a person is linked to oral health?
A) yes
B) no 